\title{
Hommage à
}
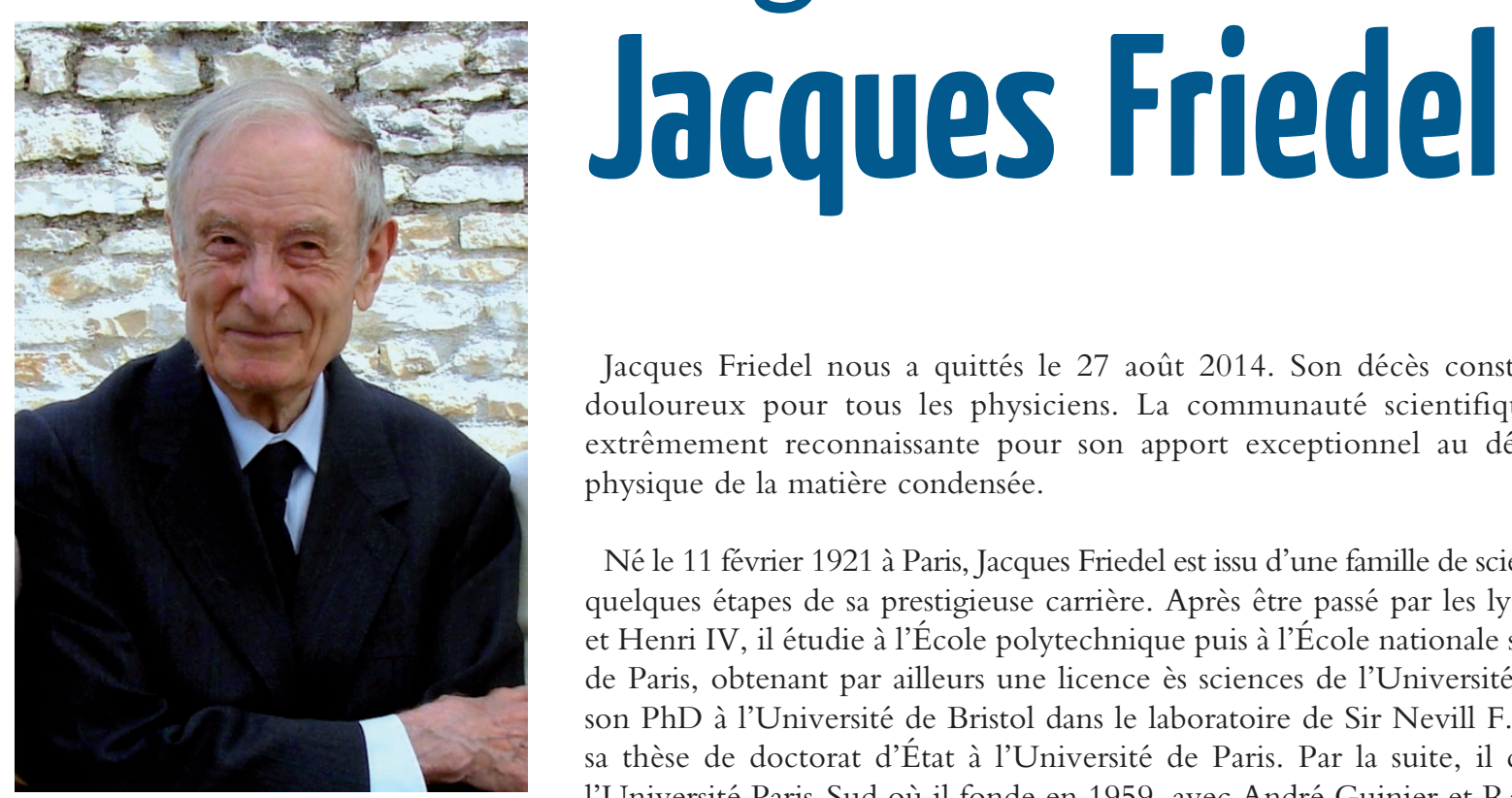

Jacques Friedel nous a quittés le 27 août 2014. Son décès constitue un événement douloureux pour tous les physiciens. La communauté scientifique française lui est extrêmement reconnaissante pour son apport exceptionnel au développement de la physique de la matière condensée.

Né le 11 février 1921 à Paris, Jacques Friedel est issu d'une famille de scientifiques. Rappelons quelques étapes de sa prestigieuse carrière. Après être passé par les lycées Louis-le-Grand et Henri IV, il étudie à l'École polytechnique puis à l'École nationale supérieure des mines de Paris, obtenant par ailleurs une licence ès sciences de l'Université de Paris. Il prépare son $\mathrm{PhD}$ à l'Université de Bristol dans le laboratoire de Sir Nevill F. Mott, puis soutient sa thèse de doctorat d'État à l'Université de Paris. Par la suite, il devient professeur à l’Université Paris-Sud où il fonde en 1959, avec André Guinier et Raymond Castaing, le Laboratoire de Physique des Solides à Orsay, qui a été et est attractif pour nombre de grands professeurs venus du monde entier pendant leurs congés sabbatiques - certains futurs prix Nobel -, ou plus jeunes scientifiques venus bénéficier d'une excellente formation dans cet environnement exceptionnel.

Jacques Friedel a été président de la Société Française de Physique (1970), de la Société Européenne de Physique (1982-1984) et de l'Académie des sciences (1992-1994). Il a aussi été élu membre associé de l'Académie Nationale des Sciences américaine, de l'Académie Royale des Sciences suédoise et de la Royal Society anglaise. Il a reçu de nombreux prix : médaille d'or du CNRS en 1970, prix Holweck, Von Hippel award... Il a enfin été élevé à la dignité de grand'croix de la Légion d'Honneur en 2013.

L'apport de Jacques Friedel à la physique de la matière condensée, en particulier à la compréhension des propriétés électroniques, a été considérable. En premier lieu, il faut citer la découverte des " oscillations de Friedel ", oscillations de densité électronique autour des impuretés dans les métaux, phénomène d'écrantage de portée très générale. Un autre aspect essentiel concerne la description des propriétés électroniques des solides par des méthodes simples : "électrons libres » et surtout "liaisons fortes ». Cette dernière, idéale pour décrire la liaison chimique, permet d'analyser, de façon très descriptive et efficace, les tendances dans les propriétés de cohésion et les excitations électroniques. Elle s'applique pratiquement à tous les types de matériaux : métaux de transition, semi-conducteurs, solides partiellement covalents et ioniques, cristallins ou amorphes... Elle est très utilisée actuellement en modélisation quantitative de composants électroniques nanométriques.

Pour terminer, ajoutons les apports de Jacques Friedel à l'analyse du rôle des défauts étendus des structures cristallisées (en particulier les dislocations, essentielles à la plasticité des métaux, auxquelles il a consacré un ouvrage en 1956, qui a connu un immense succès), à l'étude des cristaux liquides et aussi à la compréhension des différentes manifestations de la supraconductivité.

Jacques Friedel va nous manquer. Ses avis scientifiques, y compris les plus récents, ont toujours eu une très grande valeur pour nombre d'entre nous.

Michel Lannoo ${ }^{(*)}$ et Alain Fontaine ${ }^{(* *)}$

(*) Ancien président (2012-2013) de la SFP

(**) Président de la Société Française de Physique (SFP) 


\section{Présentation}

\section{Jacques Friedel, président de la SFP et de la Société européenne de physique}

Jacques Friedel ne fut pas seulement un physicien de grand talent, mais également un homme soucieux de l'intérêt général, en particulier du développement et de I'organisation de la recherche en France. Il fut ainsi amené à devenir président de la Société française de physique (SFP) en 1970, puis de la Société européenne de physique (EPS) de 1982 à 1984 ; je le connus dans ces deux fonctions.

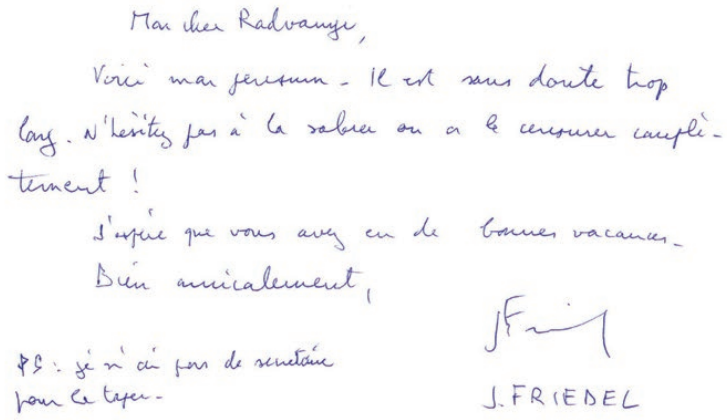

J.FRIEDEL

$[\ldots]$

Le rote noinant des regions facilite a prion l'mitiative it l'enacumement local des unversites. Il a pose dis le dejent des roblemies de qualite' th he coadinatia nationale que 6 CNRS a cherchi a réonde en accaupaynant les viditi regionaus. Mais avec be ible uorinont der viéditide

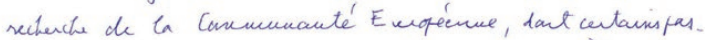
sent per les rifians, c'ent le maintien l'un aristiage national des grandes optrass qu' est en question. En Clair vent. on i aligner su le modele des Länder allemands, avec ses canfettions mais ses redondances, it ses riegáité' giogrephiques et politiques, on ras le modile ther centualinatems cistrumique, avec son fain play, ses gavis de perductuvite mais ausi ses siques de diférissernent genüral? Nour ne sommes pas

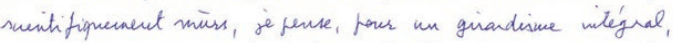
It une baune dose de jachriusme édaciè reste seus donte néés senie juign' à la fin du rieile. J.FRIESEL

Jacques Friedel s'est toujours soucié du développement et de la qualité de la recherche en France ; il s'est souvent exprimé à ce sujet dans le Bulletin de la SFP. Ci-dessus le mot d'accompagnement et la conclusion de l'article « Où va la recherche universitaire? " paru dans le Bulletin de la SFP, № 70, p. 17, octobre 1988.

Un document de 16 pages, édité par la Société Française de Physique, réunit les contributions de plusieurs des collaborateurs et élèves de Jacques Friedel, qui retracent les divers aspects de sa très riche activité scientifique. Ce document (pdf) peut être téléchargé à l'adresse : www.sfpnet.fr/hommage-a-jacques-friedel .
Tous ceux qui ont collaboré avec lui ont encore en tête - et ont peut-être conservé - ces petits mots manuscrits rapidement écrits, parfois dans un train, posant des questions ou faisant des remarques sur un problème lui tenant à cœur ; ils n'étaient jamais à négliger. On était frappé par la subtilité de son analyse et la sûreté de son jugement. Sa retenue et sa modestie étaient appréciées.

Jacques Friedel fut président de notre Société française de physique en 1970 et s'intéressa toujours à nos actions : chaque fois que la SFP souhaitait faire passer un message aux pouvoirs publics ou que lui-même sentait qu'un tel message était nécessaire, il prenait sa plume et écrivait un article significatif pour notre Bulletin. On peut ainsi suivre sa trace dans les numéros successifs.

"Laissera-t-on rouiller l'outil ? », son allocution lorsqu'il reçut la médaille d'or du CNRS est publiée en janvier 1971 ; il y relève la nécessité " du contact avec le monde de la physique appliquée, tant pour lui transmettre une connaissance utilisable que pour en tirer certains thèmes de recherche fondamentale. » Mais il ajoute : "Nous nous méfions de la tentation dirigiste en recherche fondamentale. » Il se préoccupa du recrutement des jeunes chercheurs, de l'emploi des jeunes docteurs, des moyens accordés à la recherche, ainsi que des publications de physique, notamment du Journal de physique qui se doubla d'un journal de lettres. Il accueillit dans son laboratoire le secrétariat de la Commission des publications, chargé de recevoir les manuscrits et de les envoyer aux rapporteurs.

Il prit part à l'installation de la Société européenne de physique (EPS) qui se proposait, malgré la guerre froide, de réunir les physiciens de l'Ouest et de l'Est de l'Europe. Son siège fut d'abord fixé à Genève. J. Friedel devint membre de son Comité exécutif, puis en fut le président de 1982 à 1984. Là aussi, il s'intéressa particulièrement aux publications et $s^{\prime}$ efforça d'obtenir la fixation de certaines règles. Il était préoccupé par la double structure des adhérents de I'EPS : membres individuels et sociétés nationales, dont le rôle était essentiel. Il œuvra pour l'amélioration du travail des divisions de spécialité ; la division de la matière condensée se développa, mais il ne put pas empêcher la sécession des opticiens. Il préconisa l'étude d'une source européenne de rayonnement synchrotron.

C'était instructif d'observer de la salle, l'expression de son visage quand il était assis à la tribune lors d'une réunion de l'EPS : lorsqu'il fronçait le nez, je savais qu'il était en désaccord avec ce que l'intervenant était en train de dire. Nous nous sommes ainsi retrouvés à plusieurs reprises à Genève et nous nous asseyions quelquefois à la terrasse d'un café, évoquant les questions d'actualité.

Une année, le directeur du CNRS arrivait en fin de mandat. Nous étions tous les deux assis au soleil à proximité du siège de I'EPS en attendant l'heure fixée. J. Friedel me raconta l'entrevue qu'il avait eue la veille à Paris avec le ministre et la discussion qu'ils avaient eue sur l'évolution de la recherche en France. II se tut. Je sentis alors qu'il s'était passé quelque chose de plus; je le regardai et lui dis : "Et le ministre vous a proposé de devenir directeur du CNRS et vous avez refusé ? " Il répondit : "Oui, c'est cela. », et il sourit.

Pierre Radvanyi Ancien secrétaire général de la SFP, ancien membre du comité exécutif de l'EPS 


\section{L'apport de Jacques Friedel à la physique de la matière condensée}

Jacques Friedel a joué un rôle majeur dans le développement des recherches concernant d'une part les défauts dans la matière condensée et leur impact sur les propriétés macroscopiques, et d'autre part les propriétés électroniques des solides en relation avec leur cohésion et leur structure.

Jacques Friedel débute ses travaux de recherche en 1948 au laboratoire de métallurgie de l'École des mines de Paris. La métallurgie est, à l'époque, une discipline en plein essor. Le rôle des défauts - ponctuels, linéaires, de surface ou d'interface commence à être reconnu, mais n'a fait l'objet que de peu d'études. De nouvelles techniques permettent désormais d'étudier ces défauts. C'est ainsi que l'on peut, par exemple, visualiser les dislocations (décoration par des figures d'attaque chimique, spirales de croissance des cristaux) : celles-ci ne sont plus des objets mathématiques dont l'existence et le rôle ressortent de spéculations ! Par ailleurs, d'un point de vue microscopique, les bases de la physique des solides pour les cristaux parfaits sont établies ; en témoigne le livre de F. Seitz, The modern theory of solids, publié en 1940. Elles rendent désormais possible la compréhension détaillée de la cohésion, de la stabilité de phase, des propriétés des défauts, de leur structure, de leur stabilité, de leurs interactions et de leur dynamique.

Deux voies de recherche convergentes s'ouvrent : d'une part l'étude à une échelle "mésoscopique " des défauts et de leur impact sur les propriétés macroscopiques, d'autre part l'étude à l'échelle microscopique de la structure électronique des métaux, de leur cohésion et des défauts associés. Jacques Friedel développera ses recherches selon ces deux directions, dont le rapprochement apparaît, dès cette époque, comme une nécessité.

J. Friedel rejoint donc naturellement en 1949 le H.H. Wills Physical Laboratory de Bristol, où C. Franck et N.F. Mott développent ces orientations. Il y reste trois ans et y soutient sa thèse sous la direction de N.F. Mott sur les propriétés électroniques des impuretés dans les métaux. Il revient en 1952 à l'École des mines de Paris. Il y crée un groupe de théoriciens ; celui-ci rejoint le Laboratoire de Physique des Solides d'Orsay (L.P.S.), que J. Friedel crée en 1959 avec André Guinier et Raymond Castaing. Dès lors, il anime le L.P.S. et y poursuit ses recherches avec ses élèves et collaborateurs.

Parmi les résultats marquants de J. Friedel concernant les défauts dans la matière condensée et leur impact sur les propriétés mécaniques des matériaux, je me limite ici à citer quelques-uns des premiers travaux concernant la montée, le glissement dévié, les réseaux de dislocations, leur stabilité et leur évolution sous sollicitation, le durcissement par un réseau aléatoire d'obstacles, la fracture et, plus récemment, l'étude des disinclinaisons dans les cristaux liquides. Il faut également rappeler ici le livre sur les dislocations qu'il a écrit dès 1956 [1] et qui reste un classique.

En ce qui concerne les propriétés électroniques des solides, J. Friedel introduit puis développe d'abord avec ses élèves l'étude des états d'impuretés et des propriétés des alliages métalliques dilués. Dans ses premiers travaux portant sur la structure électronique des impuretés dans les métaux [2], il utilise la théorie de la diffusion ("scattering") pour déterminer la variation de la densité électronique $\Delta \mathrm{n}(\mathrm{r})$ induite autour d'une impureté par la charge supplémentaire Z apportée par celle-ci. L'écrantage de la charge Z par la redistribution électronique $\Delta \mathrm{n}(\mathrm{r})$ doit être parfait. J. Friedel obtient alors une formule (règle de somme de Friedel) reliant $Z$ aux déphasages $\delta$ induits par le potentiel de l'impureté dans les fonctions d'onde des électrons de conduction. Il montre que $\Delta \mathrm{n}$ décroît avec la distance $\mathrm{r}$ de l'impureté comme $\mathrm{r}^{-\mathrm{d}}(\mathrm{d}=$ dimension de l'espace), en oscillant avec un nombre d'onde $2 \mathrm{k}_{\mathrm{f}}$ égal à un diamètre de la surface de Fermi (fig. 1a). À trois dimensions et avec une surface de Fermi sphérique, on a $\Delta \mathrm{n}(\mathrm{r}) \sim \mathrm{r}^{-3} \cos \left(2 \mathrm{k}_{\mathrm{f}} \mathrm{r}+\delta\right)$. Il a fallu plusieurs années pour se convaincre de la généralité et de l'importance de ces "oscillations de Friedel ». Elles sont maintenant observées directement par microscopie à effet tunnel (STM) sur une surface proche des défauts (fig. 1b), ou à l'intérieur d'un " corral " d'atomes déposés sur une surface.

Lorsqu'on introduit une impureté de métal de transition (Mn par exemple) dans un métal normal (Al, Cu...) dont les électrons de conduction sont de symétrie (s, p), les états localisés d de l'impureté interagissent faiblement avec le continuum d'états de conduction de la matrice : les électrons qui occupent ces états restent longtemps sur le site de l'impureté avant de sauter sur un site voisin. Ceci se traduit par un faible élargissement $(\Gamma)$ en énergie (via la relation d'incertitude de Heisenberg) des états d et une forte augmentation de la densité d'états sur le site de l'impureté pour des énergies voisines de celle des états d, $E_{d}$ (fig. 2). Par rapport au métal pur, l'alliage dilué est alors caractérisé par une forte augmentation des propriétés (chaleur spécifique électronique, résistivité résiduelle) dépendant de la densité d'états au niveau de Fermi $\mathrm{E}_{\mathrm{f}}$, si $\mathrm{E}_{\mathrm{d}} \sim \mathrm{E}_{\mathrm{f}}$, et par un magnétisme local de l'impureté si de plus l'interaction coulombienne entre électrons sur l'impureté est suffisante. Ce modèle très populaire de "l'état lié virtuel de Friedel " a été reformulé en 1961 par P.W. Anderson.

Les impuretés interagissent entre elles par un potentiel oscillant avec la distance comme $\Delta \mathrm{n}(\mathrm{r})$. Ces interactions sont à la base de l'ordre local dans les alliages ; elles permettent de clarifier l'origine de leur stabilité et de déterminer l'énergie des défauts ponctuels ou étendus. Si, de plus, 


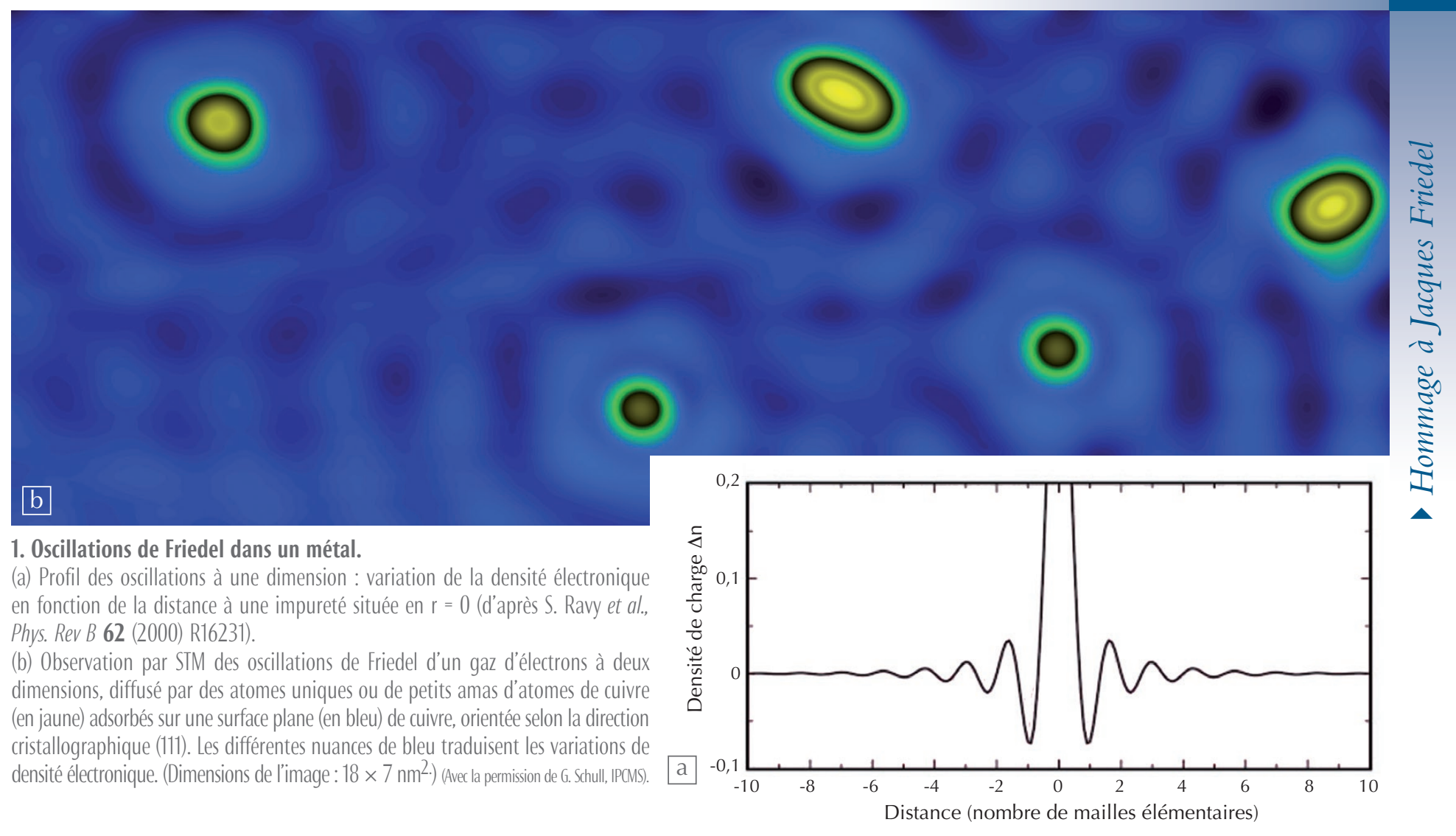

les impuretés sont magnétiques, elles interagissent magnétiquement avec un couplage oscillant. L'alliage dilué présente alors un ordre antiferromagnétique aléatoire gelé à basse température (verre de spin), qui a été introduit et étudié pour la première fois par J. Friedel et André Blandin.

Progressivement, Jacques Friedel et ses élèves ont ensuite développé au Laboratoire de Physique des Solides d'Orsay une description générale des propriétés électroniques des matériaux métalliques ou semi-conducteurs, de leur cohésion, de leurs défauts, que ces systèmes soient de

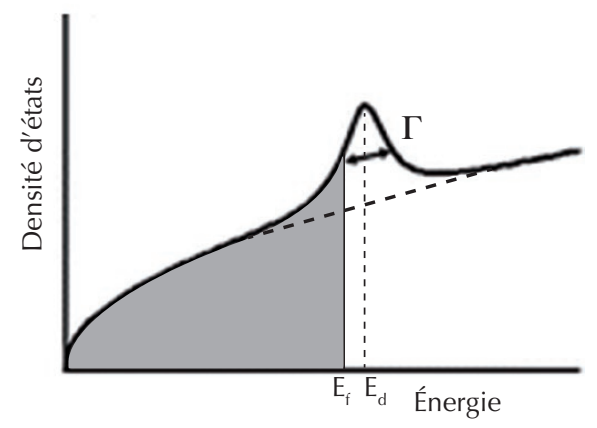

2. Niveau lié virtuel. Pic de densité d'états électroniques, de largeur en énergie $\Gamma$, associé à une impureté non magnétique de métal de transition dans un métal « normal ». $E_{d}$ : énergie du niveau atomique d de l'impureté. E Ef : énergie de Fermi de la matrice. Les états électroniques d de l'impureté sont partiellement occupés. basse dimensionnalité ou non, cristallins, amorphes ou liquides. Cette description, qui utilise l'approximation des liaisons fortes, les électrons étant dans le régime des corrélations faibles, est simple, physiquement transparente et adaptée à beaucoup de situations physiques.

Ainsi, J. Friedel et ses élèves ont montré que les propriétés spécifiques des métaux et alliages de transition, et en particulier leur magnétisme, proviennent des électrons d itinérants dans un régime de corrélations modérées. Ils obtiennent, par exemple, une interprétation physique des moments magnétiques à basse température des alliages de nickel, cobalt et fer (courbe de Pauling-Slater), en supposant que l'écran se forme essentiellement dans les bandes $\mathrm{d}$ et que, si la perturbation induite par l'impureté est trop forte dans le nickel ou le cobalt, un état lié est extrait du haut des bandes majoritaires en spin, les électrons occupant cet état étant transférés dans les bandes minoritaires. Ce modèle a permis ultérieurement de comprendre les couplages magnétiques et la magnétorésistance géante dans les super-réseaux métalliques [3].

La voie a été ainsi ouverte vers l'étude de composés à base de métaux de transition (hydrures, borures...) ou de systèmes complexes exhibant des propriétés très différentes selon leur structure atomique, la nature des orbitales mises en jeu et l'importance des corrélations. Elle est pro- longée actuellement en utilisant des méthodes plus sophistiquées, permettant éventuellement l'étude des systèmes à corrélations fortes (oxydes, par exemple).

Au contraire des électrons d des métaux de transition, les électrons $4 f$ des terres rares et de leurs composés sont localisés : leur rôle a été étudié par P.G. de Gennes. Enfin, J. Friedel a proposé une première classification des actinides, en liaison avec le remplissage et la localisation progressifs des états $5 \mathrm{f}$.

Les travaux de Jacques Friedel permettent de faire reposer sur de solides bases physiques les recherches qui se développent actuellement sur la matière condensée en utilisant des techniques numériques complexes et dans des domaines très variés, qu'il s'agisse par exemple de nanostructures en spintronique ou d'alliages multicomposants et de leur nanostructuration.

François Gautier

Professeur honoraire à l'Université de Strasbourg

\section{En savoir plus}

1 J. Friedel, Les dislocations, Gauthier Villars, Paris (1956). (2e édition en anglais, Pergamon, 1964).

2• J. Friedel, "Metallic alloys", Nuovo Cimento, 7 , Suppl. 2 (1958) 287-311.

3-A. Fert, "Les débuts de la spintronique ", Reflets de la physique 15 (2009) 5-9. 


\section{Nucléaire civil : les convictions de Jacques Friedel}

En s'intéressant aux problèmes du nucléaire civil, Jacques Friedel répétait sa conviction qu'avec la croissance démographique dans les pays en voie de développement, les besoins de nourriture et d'eau, tant pour la consommation directe que pour l'agriculture, les soins médicaux, les concentrations urbaines et suburbaines, il fallait des sources massives d'électricité. L'énergie nucléaire, une fois les problèmes cités ci-dessous réglés, lui paraissait une des sources d'énergie nécessaires. Sur son lit d'hôpital, cette réflexion sur ce monde tragique, douloureux pour tant de personnes, sur la nécessité pour qu'ils aient une vie décente d'avoir accès à l'énergie électrique, n'a pas cessé.

Dans les années 1955-1965, le déchiffrement de la métallurgie du plutonium, avec les électrons de la couche $5 f$ partiellement délocalisés à l'origine d'un diagramme de phases complexe et de propriétés physiques anormales (telle que la dilatation thermique négative de la phase $\delta$ ), nécessita un travail en profondeur. Le responsable CEA de l'époque, Emmanuel Grison, fit appel à Jacques Friedel pour le prier d'aider ses équipes. Cette collaboration allait augmenter, à mesure que les problèmes liés aux matériaux dans les réacteurs nucléaires étudiés à Saclay se révélaient cruciaux.

En 1995, le gouvernement voulut qu'un rapport soit réalisé sur l'utilité du prototype de réacteur à neutrons rapides (RNR) surgénérateur Super PheniX. Le ministre demanda à Jacques Friedel de prendre la direction d'un groupe de travail pour écrire ce rapport. J. Friedel jugea qu'il n'avait pas assez de temps pour mener cette tâche, mais assez pour y participer. Raymond Castaing accepta la tâche et créa ce groupe de travail, dans lequel Jacques Friedel s'intéressa en particulier aux propriétés mécaniques des matériaux, tant pour les structures du cœur que pour les gaines des crayons combustibles.

Après cette expérience, le projet de concept de réacteur de puissance pour les futures centrales électronucléaires qui intéressait le plus Jacques Friedel était, pour le long terme, après les réacteurs à eau pressurisée (REP) actuels et les futurs EPR (European Pressure Reactors), le surgénérateur, basé sur un spectre de neutrons (dit rapides, d'où le sigle RNR) dont le maximum se situe à une énergie de l'ordre de 0,2-0,3 MeV. À l'initiative de
Jacques Friedel et de ses collègues intéressés, plusieurs colloques de comparaison des phénomènes de matériaux advenant dans ces RNR ont été tenus à l'Académie des sciences. L'objectif de Jacques Friedel, Yves Bréchet, Georges Martin, André Pineau et moi-même, en coorganisant et menant ces colloques, était que les jeunes scientifiques et techniciens disposent d'outils synthétiques pour saisir des champs de connaissance vastes et compliqués. Le souci majeur de Jacques Friedel était l'augmentation des ressources utilisables de noyaux fissiles, grâce à un concept d'économie des neutrons favorisant leur capture par des noyaux fertiles. C'est le trait essentiel des surgénérateurs (voir encadré). Pour tirer le maximum d'énergie thermique de chaque unité de volume du cœur, il faut augmenter le flux de neutrons. Mais la puissance thermique produite par les fissions est limitée par la possibilité de l'extraire du cœur par un fluide caloporteur. Pour avoir un rendement de Carnot aussi élevé que possible, au-delà des 34\% des REP actuels, il faut que la température de

la source de chaleur soit la plus élevée possible. Dans les REP, la limite de température est donnée par la condition de ne pas laisser le fluide caloporteur bouillir, soit $350{ }^{\circ} \mathrm{C}$ à une pression d'eau de 140 bars.

Les fluides caloporteurs envisagés pour les RNR (sodium, plomb liquide, hélium, sels fondus de fluor) permettraient d'atteindre 550 à $600^{\circ} \mathrm{C}$, visant des rendements thermodynamiques de près de $40 \%$. Les accidents ont notamment illustré les limites physiques des matériaux des gaines. Jacques Friedel et al. organisèrent à l'Académie des sciences un colloque sur les fluides caloporteurs possibles pour les RNR [1]. Pour ces derniers, plusieurs fluides ainsi que différents matériaux des circuits (dont les gaines des éléments combustibles) qui les contiennent, sont envisagés.

Il reste à concevoir et développer les matériaux capables de supporter les irradiations à de hauts flux de neutrons, ces températures, les contraintes mécaniques engendrées par les gradients de source et d'évacuation de la chaleur. La fragilisation sous irradiation des aciers des cuves de réacteurs des deuxième et troisième générations est déjà un facteur limitant leur durée de fonctionnement. Ces réflexions sur les matériaux furent menées à l'Académie des sciences (réf. [2], pp. 499-500). Jacques Friedel y déploya sa profonde compréhension des mécanismes à l'œuvre dans les conditions physiques citées ci-dessus.

\section{Les surgénérateurs}

Les surgénérateurs sont des réacteurs qui produisent plus de matière fissile qu'ils n'en consomment. Dans les REP et EPR classiques, on n'utilise que les 0,72\% d'uranium 235 fissile contenu dans I'uranium naturel. Dans la filière uranium/plutonium, la surgénération vise à tirer profit des 99,28\% d'uranium 238 non fissile mais "fertile ", la capture de neutrons par $\mathrm{U}^{238}$ conduisant au plutonium 239 fissile. Les lois de la neutronique montrent que cela est possible, à condition :

- d'utiliser un combustible fait de $\mathrm{Pu}^{239}$ fissile et d' $\mathrm{U}^{238}$ fertile en proportions convenables ;

- de ne pas ralentir les neutrons avec un modérateur, c'est-à-dire d'utiliser des neutrons rapides. Les surgénérateurs produisent donc de l'électricité tout en consommant I' $U^{238}$, ainsi que le $\mathrm{Pu}^{239}$ récupéré dans le retraitement des combustibles usés des réacteurs REP. Ceci permettrait de gagner un facteur 40 à 50 sur les ressources de matière fissile disponibles. 
On sait que les noyaux radioactifs à demi-vie longue formés dans les cœurs des réacteurs posent problème pour leur stockage définitif, par exemple en couche géologique souterraine. L'un des degrés de liberté du système de stockage est la capacité d'un conteneur de substances radioactives à être totalement étanche pendant de très longues durées, ce qui est un problème de matériaux que Jacques Friedel et al. étudièrent (réf. [2], pp. 489-490). Le rôle des divers isotopes du plutonium dans le cycle du combustible en France a préoccupé Jacques Friedel et al. [3]. Quel sort donner aux actinides produits durant l'irradiation des éléments combustibles et conduisant à l'accumulation des isotopes de l'américium, du curium et du neptunium (isotope $\mathrm{Np}^{237}$, de demi-vie $210^{6}$ ans) ? La solution de les irradier en réacteur dans des cibles, pour les réduire à des produits de fission à demi-vie courte, exige de disposer de matériaux capables de supporter, outre les conditions extrêmes du cœur déjà citées, les pressions internes à ces cibles engendrées par les gaz de fission, les particules $\alpha$ et les fissions spontanées, toujours présents, même quand la cible est sortie du cœur du réacteur.

Une question lancinante des entretiens de Jacques Friedel et al. a été de s'interroger sur la possibilité de comparer entre eux les risques des grands systèmes technologiques, et notamment, pour le nucléaire civil, des accidents majeurs dispersant des substances radioactives dans de vastes régions, dans l'atmosphère, dans les eaux tant du sol que météoriques, et cela pendant des durées indéfinies. S'agit-il d'une propriété du nucléaire civil, ou bien d'une caractéristique des concepts actuels de réacteurs basés sur des combustibles solides ? Quels sont les moyens d'agir sur ces grands systèmes nucléaires pour minimiser leurs risques et leurs conséquences?

Nous avons observé que le fait le plus important du nucléaire civil depuis son début dans les années 1950 est que les réacteurs de fission, modérés et refroidis à l'eau ordinaire, sont devenus le concept international utilisé par la majorité des

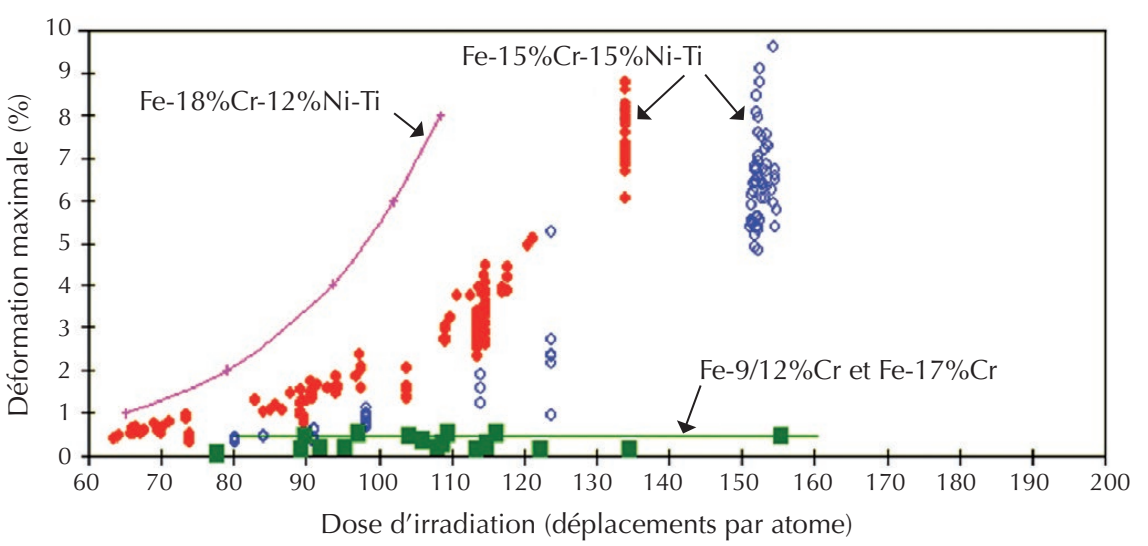

Déformation de gonflement en fonction de la dose pour plusieurs aciers irradiés aux neutrons rapides. Les compositions des aciers sont indiquées sur la figure. (D’après J.L. Séran, communication personnelle.)

entreprises de production d'électricité. Ceci est dû largement aux qualités physiques, chimiques et mécaniques des aciers et des alliages de zirconium en contact avec les fluides caloporteurs.

Nous avons constaté que tous les combustibles utilisés dans les centrales électronucléaires actuellement en fonctionnement sont en matériau solide. On ne peut donc les sortir du réacteur qu'à de très longs intervalles se comptant en années, pendant lesquelles ils deviennent des agrégats de produits de fission et d'actinides radioactifs mélangés, sources de chaleur et de substances radioactives gazeuses ou volatiles pouvant être dispersées en cas de défaillance des gaines. Peut-on faire progresser la sureté nucléaire par une modification de ce paradigme de la physico-chimie des installations nucléaires ? Un exemple, avec un combustible fluide, en est donné dans la référence [4].

C'est en pesant chaque mot que Jacques Friedel et al. ont écrit (réf. [2], p. 509) : "Dans la perspective de centrales électronucléaires construites dans le monde entier, on peut se demander si les préoccupations concernant l'emploi présent et futur de l'énergie électronucléaire sont justifiées. L'une d'elles est inéluctable... C'est que toute centrale électronucléaire (avec les installations associées du cycle de combustible), où qu'elle soit située dans le monde, ne devrait impliquer que des personnels expérimentés, munis des équipements en état de marche, toujours prêts à intervenir à tout moment, en cas d'événements naturels, de bouleversement politique ou belliqueux, ou de troubles des grands systèmes. Une telle surveillance devrait être maintenue, pendant le fonctionnement... et tant que les éléments combustibles irradiés sont dans le bâtiment du réacteur. L'électricien français et l'autorité de sûreté ont assumé, jusqu'ici, tous les aspects de cette tâche rigoureuse et vigilante. "

Robert Dautray

Membre de l'Académie des sciences Ancien Haut-Commissaire à l'énergie atomique

\section{En savoir plus}

1- Fluides caloporteurs pour réacteurs à neutrons rapides, Académie des sciences, EDP Sciences (2014).

2• R. Dautray, J. Friedel et Y. Bréchet, « Réflexions sur l'avenir de l'énergie nucléaire, de la France d'aujourd'hui au monde de demain : II à IVe générations", C.R. Physique 13 (2012) 480-518.

3• « Le plutonium et la cohérence du parc électronucléaire français ", C.R. Physique 14 (2013) 228-247.

$4 \bullet$ « Contrôler et limiter la dispersion des produits radioactifs des centrales électronucléaires en cas d'accident », C.R. Physique 15 (2014) 481-508. 


\section{Jacques Friedel, la métallurgie et l'industrie}

La métallurgie traverse toute la carrière de Jacques Friedel, depuis ses tout premiers travaux sur l'énergie des joints de grain, effectués dans le laboratoire de son cousin C. Crussard, ou sa thèse avec N.F. Mott sur les états liés virtuels, jusqu'à ses dernières réflexions sur la dynamique du frottement entre plaques tectoniques, et sur la structure électronique des quasicristaux.

J. Friedel est au tout premier rang des fondateurs de la discipline, puis parmi ceux qui portent la science des matériaux, héritière de la métallurgie, et enfin parmi ces scientifiques qui s'intéressent presque instinctivement aux applications industrielles de leur domaine, au nombre desquels sont des noms aussi prestigieux que Kelvin, Pasteur ou de Gennes.

\section{Le siècle d'or de la métallurgie : les pères fondateurs}

Jacques Friedel était l'incarnation de la métallurgie "moderne ». Longtemps empirique, la métallurgie s'est constituée comme véritable science dans les années 1930-60, période où ont émergé les concepts fondateurs de la discipline et les grands noms qui les ont portés :

- la théorie des défauts avec G.I. Taylor,

E. Orowan, J. Eshelby, N.F. Mott,

F.R.N. Nabarro, P. Haasen, A. Seeger,

A. Cottrell et J. Friedel ;

- la théorie électronique de la cohésion des solides, avec encore N.F. Mott, J. Friedel, A. Cottrell, W. Kohn ;

- la théorie des transformations de phases, avec C. Zener, D. Turnbull, J.W. Cahn, M. Hillert, J.W. Christian, W. Mullins, M. Flemings.

Deux générations ont ainsi construit la discipline en tant que science théorique, pendant qu'en parallèle, une floraison de méthodes expérimentales nouvelles sont apparues :

- microscopie électronique, avec P.B. Hirsh,

- diffusion centrale des rayons X puis des neutrons, avec A. Guinier,

- analyse chimique locale, avec R. Castaing.

Ces méthodes ont apporté une moisson de résultats qui ont fondé solidement la volonté de cette métallurgie nouvelle de comprendre les propriétés des métaux et alliages à partir d'une compréhension fine des microstructures, et de comprendre l'influence des procédés de transformations via la genèse des microstructures.
On a pu dire avec quelque apparence de raison qu'en lisant attentivement les premiers volumes de la revue Acta Metallurgica entre 1958 et 1968, on avait l'essentiel des idées en place, dans leur lumineuse simplicité, et que les deux générations suivantes ont essentiellement écrit des notes de bas de page des travaux de ces géants, dont au tout premier rang, J. Friedel et son jumeau britannique A. Cottrell ${ }^{(a)}$.

La génération suivante, celle des élèves de Friedel en France, a permis le plein développement de ces idées et de ces méthodes, et a en quelque sorte irrigué le territoire académique français : Poitiers, Lille, Lyon, Nancy, Saint-Étienne, Grenoble... Dans chacune de ces villes on peut sans difficulté construire une généalogie scientifique qui, immanquablement, remonte à Friedel. On ne compte plus les témoignages de ceux qui, dans les années 1970, ont pu monter des groupes de recherche grâce aux "actions thématiques programmées " que Friedel orientait judicieusement.

Il ne fut pas très favorable au calcul numérique intensif en métallurgie, son goût le portant naturellement vers les "petits modèles simples ". Mais au travers de ses visites régulières au laboratoire de Y. Adda puis G. Martin (SRMP, CEA/ Saclay), il en suivait les développements, et les guidait par ses questions touchant directement le cœur des problèmes, au-delà des images séduisantes. Il y avait du calviniste dans ce regard critique sur «l'iconographie numérique ".

\section{Faire sortir la métallurgie de son ghetto: vers une science des matériaux}

L'examen de la longue et riche carrière de Jacques Friedel montre à quel point il était conscient que la vraie place de la métallurgie était au cœur de la science des matériaux. Les concepts développés pour les métaux, en particulier ceux des défauts topologiques, s'avèrent très fertile dans d'autres domaines. Rejoignant en cela C. Franck et P. Haasen, il s'est intéressé aux dislocations et disinclinaisons dans les cristaux liquides, aux concepts de plasticité des ondes de densité de charge (où, comme pour les réseaux de vortex dans les supraconducteurs, les dislocations jouent un rôle clé dans la compréhension des courants critiques).

Dans ses toutes dernières années, il travaillait sur la plasticité des métaux nanostructurés. Il n'a jamais cessé de s'intéresser aux structures électroniques, des métaux et alliages en général, mais aussi des agrégats, des quasicristaux, des supraconducteurs à haute température. Une anecdote pour montrer l'étendue de sa curiosité : présidant le groupe de travail de l'Académie des sciences sur les aspects séismiques de l'accident de Fukushima (à plus de 90 ans, il y voyait " une occasion d'apprendre quelque chose "), il faisait part dans sa correspondance de quelques idées simples pour estimer l'amplitude du glissement des plaques tectoniques, s'appuyant sur les concepts de mouvements des dislocations, qu'il avait forgés pour de tout autres questions. 

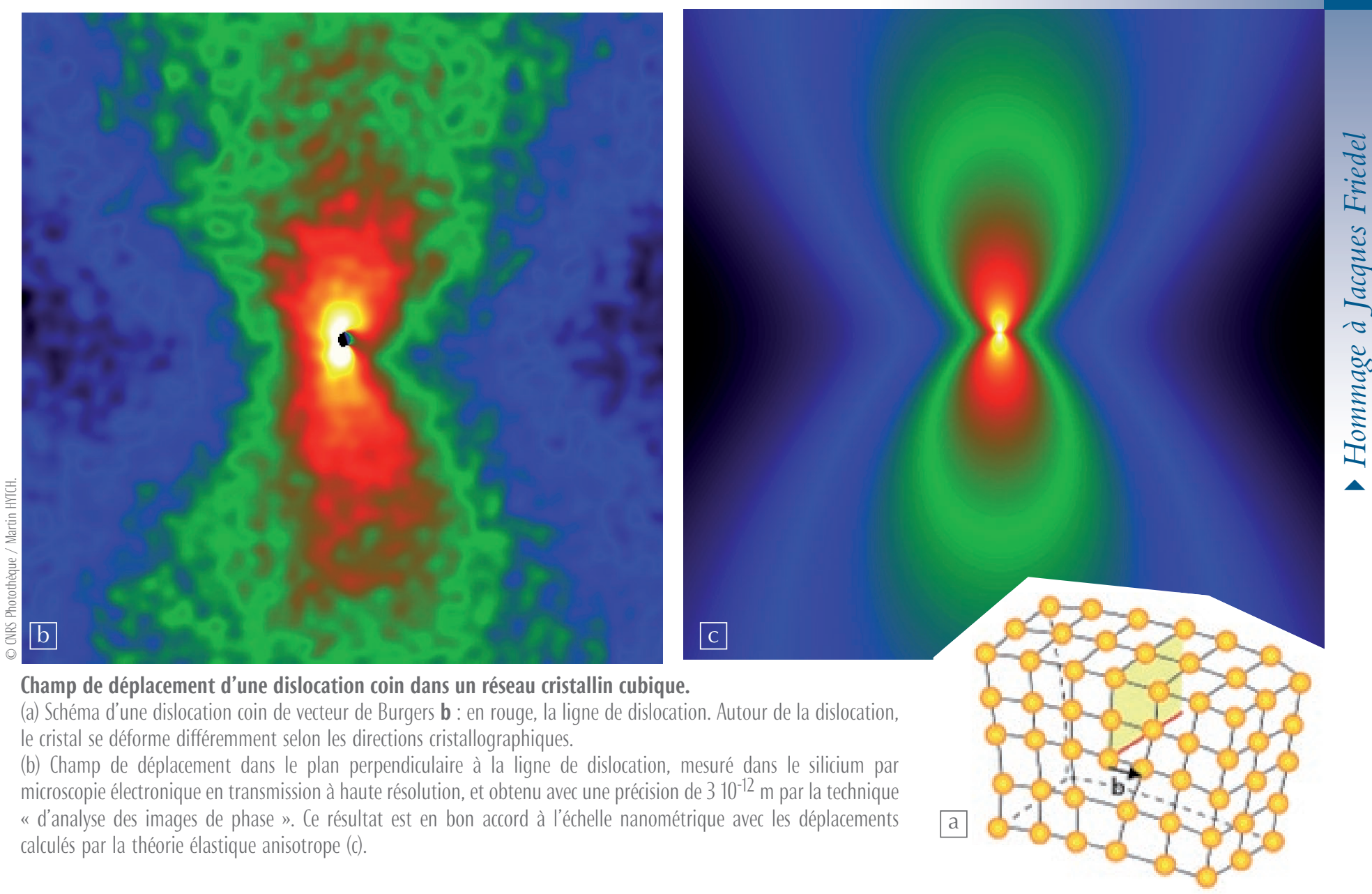

Champ de déplacement d'une dislocation coin dans un réseau cristallin cubique.

(a) Schéma d'une dislocation coin de vecteur de Burgers $\mathbf{b}$ : en rouge, la ligne de dislocation. Autour de la dislocation, le cristal se déforme différemment selon les directions cristallographiques.

(b) Champ de déplacement dans le plan perpendiculaire à la ligne de dislocation, mesuré dans le silicium par microscopie électronique en transmission à haute résolution, et obtenu avec une précision de $310^{-12} \mathrm{~m}$ par la technique " d'analyse des images de phase ». Ce résultat est en bon accord à l'échelle nanométrique avec les déplacements calculés par la théorie élastique anisotrope (c).

\section{La métallurgie et l'industrie : une inquiétude militante}

Jacques Friedel est issu d'une dynastie de scientifiques. Il est aussi apparenté à une dynastie d'industriels. Il n'a jamais oublié que la métallurgie est une science mais aussi une industrie, et que cette industrie ne sera innovante et compétitive que si elle sait s'approprier et soutenir la recherche de base. Peu de gens ont eu comme lui cette intuition des problèmes fondamentaux posés par les questions industrielles, et des conséquences appliquées des développements fondamentaux. Dans le domaine de la métallurgie nucléaire qui l'a toujours intéressé, il voyait à la fois ce que la physique fondamentale des solides pouvait permettre de comprendre sur la chimie des actinides, mais aussi la richesse des comportements sous irradiation des populations de défauts. En ce sens le parallèle avec de Gennes dans le domaine de la « matière molle " vient de suite à l'esprit : irriguer d'un double flux la science et l'industrie, chacun continuant à faire son métier dans le respect de celui de l'autre. En des temps de programmation tatillonne, la leçon mérite d'être méditée.

Il était de la génération pour laquelle le centre de recherche de la sidérurgie (IRSID) à Saint-Germain-en-Laye avait été une école de problèmes fondamentaux. Combien se souviennent aujourd'hui de ce beau papier qu'il écrivit sur les instabilités des interfaces métal-laitier ? Jacques Friedel n'avait de cesse de critiquer le désengagement de l'industrie française de la recherche fondamentale en métallurgie, et il y voyait un risque grave pour notre pays... L'avenir lui a hélas donné raison et, au moment où on parle de réindustrialisation, on ferait bien de se souvenir de cette grande voix. Il n'était pas plus tendre pour Philips ou France Télécom. C'est qu'il y avait du " grand commis de l'État " dans ce Professeur, un engagement fort au service du pays, par des rapports toujours incisifs ${ }^{(b)}$.

Il avait vivement encouragé la rédaction du rapport conjoint de l'Académie des sciences et de l'Académie des technologies sur la métallurgie ${ }^{(c)}$, il en a suivi la rédaction, il en a fait la lecture critique et l'a présenté à l'Académie des sciences en personne. On ne peut mieux faire pour conclure que de lui laisser la parole...

Yves Bréchet

Membre de l'Académie des sciences

"Il faut bien comprendre au contraire que ce qui est demandé est un changement d'attitude vers le long terme et des mesures initiales qui touchent plus à l'organisation de ce qui existe qu'à des dépenses supplémentaires importantes. [...] La crise actuelle n'est pas plus importante que celle des années 30. Or c'est dans les années 30 que les firmes sidérurgiques françaises ont décidé de créer un laboratoire commun qui deviendra l'IRSID [...]. Le décret Suchet en 1939 faisait obligation à tous les corps de l'État d'affecter à la recherche 10\% de leur personnel. Cette mesure simple et peu con̂teuse a été un facteur positif pour le renouveau de la recherche après-guerre, notamment en métallurgie. "

Jacques Friedel

(a) Il ne faut, en aucun cas, voir dans cette phrase une dépréciation des successeurs, mais plutôt un écho du mot de Bertrand Russell, disant que toute la philosophie se bornait à une série de notes en bas de page d'une édition de Platon : les bonnes questions sont posées dès l'origine, permettant des développements fructueux.

(b) Celui rendu à Raymond Barre en 1980 sur la recherche gagnerait à être relu aujourd'hui, il n'a pas pris une ride...

(c) La métallurgie, science et ingénierie (2011). La présentation du rapport à l'Académie par J. Friedel est un modèle du genre : vision cohérente de la recherche, de l'enseignement et de l'industrie, réflexions sur le passé, vue d'ensemble de la discipline et de ses ramifications, recommandations pour l'avenir. Voir aussi Reflets de la physique 24 (2011) 16-20. 


\section{Plasticité ou fragilité de la recherche française en physique?}

Nous publions ci-dessous un texte de Jacques Friedel, paru en mars 2004 dans le numéro 143, page 31, du Bulletin de la Société Française de Physique, à la suite de la crise grave de financement de la recherche de 2003-2004. On ne peut qu'être frappé par la justesse et l'actualité du propos, plus de dix ans après sa publication.

La rédaction
Lancée par des patrons des sciences de la vie [Appel de Cochin, 4 avril 2003], la protestation récente concernant les crédits de recherche dénote un mécontentement plus général. Dans un contexte certes difficile, le budget de la recherche semble géré de façon assez chaotique, peu favorable aux initiatives au niveau des laboratoires. L'exemple de la physique montre que les problèmes sont profonds et généraux. Ils méritent une réflexion à long terme, pour laquelle une réunion style colloque de Caen 1956 ne peut être utile que si elle est largement préparée et suivie d'autres initiatives. En fait, la crise de la recherche reflète la crise de notre société.

Trois facteurs se conjuguent en effet, comme nous le voyons bien en physique : démographique, économique et culturel.

Démographie : l'afflux des jeunes, les besoins de la reconstruction, le développement des villes aux dépens de la campagne, ont produit après la dernière guerre un accroissement considérable du monde universitaire, comme de la recherche. Le développement, plus tardif en France que dans les pays anglo-saxons, produit la vague de retraites actuelle. Et le volume et l'intensité de la recherche vont être difficiles à maintenir globalement, du simple fait d'une natalité française défaillante. La situation sera encore plus tardive et catastrophique en Allemagne. Mais même aux États-Unis, les seules solutions, en sciences pour l'ingénieur (SPI) et en physique, sont la formation et l'utilisation majoritaires de chercheurs étrangers, essentiellement d'Extrême-Orient, et plus récemment la délocalisation partielle du développement et même de la recherche dans ces mêmes pays.

Économie : le deuxième facteur négatif provient d'un désinvestissement progressif dans la recherche industrielle. Du fait, entre autres, que les revenus industriels sont à la base d'une fraction importante des fonds de retraite, les industries dépendent de plus en plus d'actionnaires qui exigent des revenus annuels importants et garantis. Ceci privilégie les directeurs financiers plutôt que les techniciens, et l'achat ou la vente de firmes par appartements plutôt que le maintien d'une recherche et d'un développement à long terme. À l'extrême, les laboratoires industriels sont remplacés par des contrats avec des PME que l'on soutient en partie, souvent délocalisées dans les pays du tiers-monde. À cette tendance générale s'ajoute, pour la physique et un certain nombre de SPI, une diminution de l'effort de recherche dans les secteurs nationalisés en cours de dénationalisation. De même, dans le secteur de la défense nationale, l'évolution rapide des problèmes à résoudre et des techniques est un facteur d'instabilité.

Culture scientifique : l'évolution des modes d'information scientifique conduit à une domination de fait de la recherche anglo-saxonne. Ainsi, pour les publications, leurs journaux privés, dont les politiques sont loin d'être neutres, sont considérés par les jeunes chercheurs, en physique comme ailleurs, non seulement comme le nec plus ultra, mais en fait comme la seule référence valable. Les communications par courriel et les index de citation complètent actuellement un effort - peut-être inconscient - d'imposer trop tôt un credo unique sur les problèmes scientifiques pendants. De même, toute réflexion prospective sérieuse par les chercheurs eux-mêmes semble le plus souvent abandonnée à l'échelle nationale ou même européenne, à l'exception de quelques grandes machines de hautes énergies ou d'astrophysique, ou de secteurs comme l'espace ou la fusion nucléaire où l'Europe semble actuellement distancée, pour des raisons peut-être plus politiques que scientifiques. 


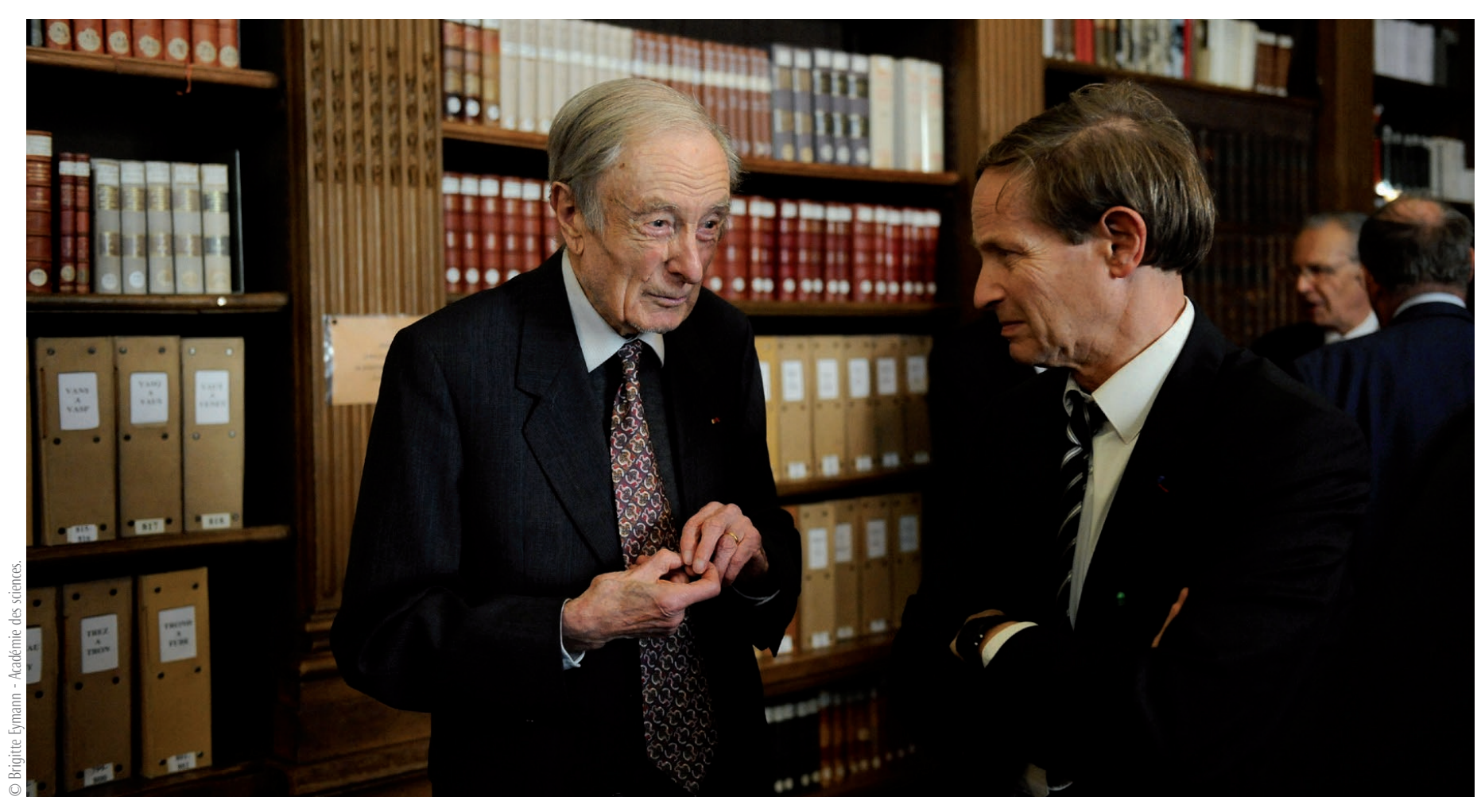

Jacques Friedel avec Sébastien Candel, actuellement vice-président de l’Académie des sciences.

Que faire ? On peut penser que ces problèmes rendent la situation éminemment plastique : il devient beaucoup plus facile de circonscrire les citadelles vieillissantes; il devient concevable d'éteindre de larges secteurs considérés comme endormis, pour développer les secteurs d'avenir. C'est en fait ce qui a été répété depuis deux ans au moins : il faudrait se concentrer sur quelques domaines - sciences de la vie, mathématiques appliquées, environnement... - imitant les Anglo-saxons, il faudrait stimuler les jeunes chercheurs en privilégiant les petits groupes productifs et pas chers, menés par des chefs de projet recrutés pour un temps limité, ayant si possible un pied - ou les deux - dans l'industrie.

Mais en France, la recherche semble trop fragile pour ce jeu. Ainsi, à force d'affirmer que, des sciences, seules celles du vivant avaient un avenir, mais en contingentant en même temps la formation médicale sans prévoir de places claires pour les futurs chercheurs, on a convaincu l'ensemble des jeunes de se détourner de toute formation scientifique universitaire. Sans cette pression à la base, toutes les filières scientifiques universitaires, biologie comprise, voient naturellement leurs postes libérés par les retraites s'évaporer au profit d'autres secteurs, moins porteurs encore de débouchés.

Dans ce passage délicat de relais entre générations, les nouveaux retraités n'ont pas à rougir de leur action depuis les années 1960. Mais c'est à la nouvelle génération de prendre sa destinée en main, avec modestie mais détermination. Elle doit avoir conscience de l'importance d'entretenir en France des foyers de recherche pertinents et originaux, qui contribuent à la formation des élites comme au progrès des connaissances. Ceci ne se fait pas en un jour et demande un effort constant et sérieux de prospective scientifique et technique, tant au niveau national qu'européen. Un dialogue doit être mené avec l'industrie sur la place que doivent conserver la recherche et l'innovation industrielle en France. Enfin, toute réforme profonde d'organismes comme le CNRS devrait être précédée par une révision du système universitaire, qui donne sa place à une recherche fondamentale de pointe, mais aussi à des activités d'innovation technologique plus proches de l'environnement local.

Il est clair que toute réforme ne peut être uniquement parachutée d'en haut. En principe, des contextes comme la Société Française de Physique et l'European Physical Society devraient jouer un rôle important dans les réflexions.

Jacques Friedel 